EASD

Procedia
EURODYN 2020 XI International Conference on Structural Dynamics M. Papadrakakis, M. Fragiadakis, C. Papadimitriou (eds.) Athens, Greece, 23-26 November 2020

\title{
DYNAMIC EFFECT OF TRAINS WITH ARTICULATED COACHES AND JACOBS BOGIES WITH INTEGER WHEELBASE RATIOS
}

\author{
Pedro Museros $^{1}$, Andreas Andersson ${ }^{2}$, and Raid Karoumi ${ }^{2}$ \\ ${ }^{1}$ Universitat Politècnica de València \\ Camino de Vera s/n, 46022 València, Spain \\ e-mail: pmuseros@mes.upv.es \\ ${ }^{2}$ Royal Institute of Technology \\ Brinellvägen 23, SE-100 44 Stockholm \\ e-mail: adde@kth.se; raidk@kth.se
}

Keywords: Railway bridge, high-speed train, bogie wheelbase, train spectrum.

\begin{abstract}
This paper deals with the dynamic response of railway bridges traversed by articulated trains, specifically the interaction effect between the coach length $D$ and bogie distance $d_{B A}$. Current design code EN 1991-2 stipulate that the ratio $D / d_{B A}$ should not be close to an integer value, but no further guidance is given. By using train signatures and a so-called bogie factor, the combined effect is illustrated in a non-dimensional domain. The results are also presented by realistic examples which show that integer ratios of $D / d_{B A}$ are not necessarily worse than non-integer ratios. This is further confirmed by a parametric study of simply supported beams and ranges of train parameters according to EN 1991-2 Annex E.
\end{abstract}

ISSN:2311-9020 @ 2020 The Authors. Published by EASD Procedia.

Peer-review under responsibility of the Organizing Committee of EURODYN 2020. doi: $10.47964 / 1120.9217 .20270$ 


\section{INTRODUCTION}

Excessive vibrations may occur in railway bridges if the load frequency coincides with the natural frequency of the bridge. In the design of railway bridges, especially on high-speed lines, the dynamic response is analysed using a set of universal trains, named as HSLM in EN 1991-2 [1]. These train load models were developed within ERRI D214.2/RP6 [2] and should also cover ranges for different real trains, as defined in EN 1991-2 Annex E. Excessive vibrations are likely to occur at resonance, where the load frequency depends on the axle spacings or any of its integer multiples.

This paper focus specifically on the articulated train (AT) with jacobs bogies, where the coach length $D$ and bogie distance $d_{B A}$ are the main parameters. In EN 1991-2 Annex E it is stipulated that $D / d_{B A}$ should not be close to an integer value. No further guidance is given on how close to an integer value such ratio is allowed, or how to assess its effect. The main aim of this study is therefore to explore the interaction effect between $D$ and $d_{B A}$ in terms of the resulting dynamic response. This is done by a parametric study of simply supported bridges and a non-dimensional approach based on train signatures and a so-called bogie factor, further explained in this paper.

\section{MOTIVATION OF THE RESEARCH}

A typical example is presented first, in order to motivate the reasons for this investigation. Let a theoretical simply supported (S-S) railway bridge have first natural frequency $n_{0}=7.0 \mathrm{~Hz}$, which is a usual value for spans between some 12.5 and $30 \mathrm{~m}$. Consider also an AT (see Figure 1) with characteristic distances $D=18 \mathrm{~m}$ (carriage length) and $d_{B A}=b=3 \mathrm{~m}$ (bogie wheelbase) travelling over the bridge. For the moment, the presence of the power cars (1) in Figure 1 is ignored, and it is admitted that all passenger coaches of type (2) and (3) have the same distances $D$ and $b$. These hypotheses allow one to focus on the possible resonant coupling caused by integer values of the $D / b$ ratio.

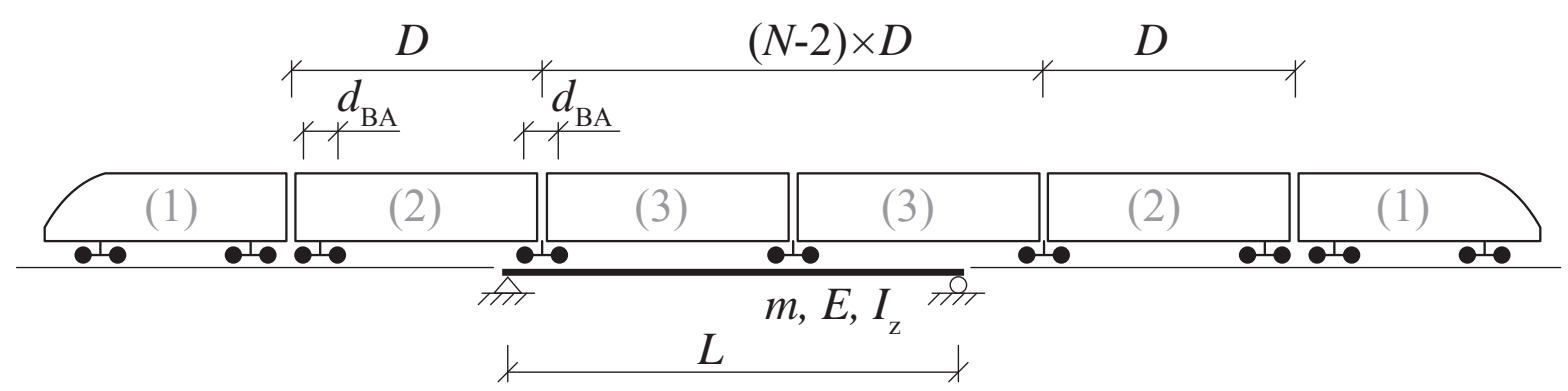

Figure 1: Articulated train passing over a S-S bridge.

Any mode of vibration of the bridge that is excited by the passing train will remain in free vibration once the train has left the bridge. The passage of each axle load generates such kind of free vibrations, that will accumulate with the free vibrations generated by the subsequent passing loads, in a way that may lead to (i) resonance in the case of in-phase addition, (ii) cancellation if the addition is in counter-phase, or (iii) addition to some intermediate degree in the rest of cases. If damping were absent, which is only possible from a theoretical point of view, resonance and cancellation phenomena would be ideally perfect for axle loads of equal 
intensity adding in-phase or counter-phase, respectively. All these aspects were dealt with in depth in previous research works [3].

The relevance of an eventual integer value of $D / b$ is apparent: in a two-axle bogie, if the vibrations of the second axle add in phase with those of the first axle, this means that the time interval $b / V$, where $V$ is the speed of the train, is an integer multiple of the period $T$ of the vibration mode of interest. For the fundamental mode, this entails

$$
b / V=n / n_{0} \quad n=1,2,3, \ldots
$$

In equation (1) $n=1$ is referred to as the first bogie resonance, while for $n=2,3, \ldots$ one speaks of second resonance, third resonance, etc. With the values presented above, one gets

$$
V_{n=1}=\frac{b n_{0}}{1}=\frac{3 \cdot 7}{1}=21 \mathrm{~m} / \mathrm{s}=75.6 \mathrm{~km} / \mathrm{h}
$$

It is apparent that for speeds equal to integer fractions of $75.6 \mathrm{~km} / \mathrm{h}$, one will get the second, third, etc. resonances of the bogie. Because of damping, the amplitude of the resulting vibration from each of them will decrease as $n$ increases. Moreover, speeds around $75.6 \mathrm{~km} / \mathrm{h}$ are nowadays considered low speeds, and particular bridge vibration problems are not expected in regular operation of railway vehicles at such speeds.

In the sample case presented here, when the first resonance of the bogie takes place, then also a sixth resonance of the carriage takes place, because, in a way analogous to equation (2), one has, for characteristic distance $D=18 \mathrm{~m}$ :

$$
V_{n=6}=\frac{D n_{0}}{6}=\frac{(6 \cdot 3) \cdot 7}{6}=21 \mathrm{~m} / \mathrm{s}=75.6 \mathrm{~km} / \mathrm{h}
$$

Of course, if damping were high enough, such sixth sub-harmonic of the carriage resonance would have little effect, but it should also be born in mind that damping can be higher or lower depending on the bridge type and condition.

For free vibrations to accumulate, cancellation of the vibration mode (for each axle) must not take place [4], [3]. Therefore, the nondimensional speed $K$ must be different from $1 /(2 i+1)$ if the bridge is $\mathrm{S}-\mathrm{S}$, of span equal to $L$. Integer $i$ is referred to as the order of the cancellation of the influence line, since it yields the order of the corresponding zeros of the free vibration amplitude response (see figure 2 in reference [3]). Consequently, the condition to be verified to avoid cancellation is as follows:

$$
K=\frac{V}{2 n_{0} L} \neq \frac{1}{2 i+1} \quad i=1,2, \ldots
$$

With the reference data employed above, cancellation would happen at $75.6 \mathrm{~km} / \mathrm{h}$ for the spans listed in Table 1 . So, one can select a span equal to $L=15 \mathrm{~m}$ and be sure that is neatly far from cancellation, and indeed very close to a point of maximum free vibration. In such case, the addition of successive axle loads will lead to a maximum resonance phenomenon (see section 4 in reference [3]).

Figure 2 shows the response of the above described bridge under the circulation of a series of $2 k=20$ loads, arranged in $k=10$ equidistant bogies with two loads $P=170 \mathrm{kN}$ in each bogie. The linear mass of the bridge is set to $m=12 \mathrm{t} / \mathrm{m}$, which is a realistic value for a concrete, single-track, beam bridge of $15 \mathrm{~m}$ span, and damping ratio is $\zeta=1.0 \%$. Damping 


\begin{tabular}{c|ccccccc}
\hline$i$ & 1 & 2 & 3 & 4 & 5 & 6 & 7 \\
\hline$L(\mathrm{~m})$ & 4.5 & 7.5 & 10.5 & 13.5 & 16.5 & 19.5 & 22.5 \\
\hline
\end{tabular}

Table 1: Span lengths for $i$-th cancellation of the $1^{\text {st }}$ mode at $75.6 \mathrm{~km} / \mathrm{h}$ in a S-S bridge with $n_{0}=7.0 \mathrm{~Hz}$.

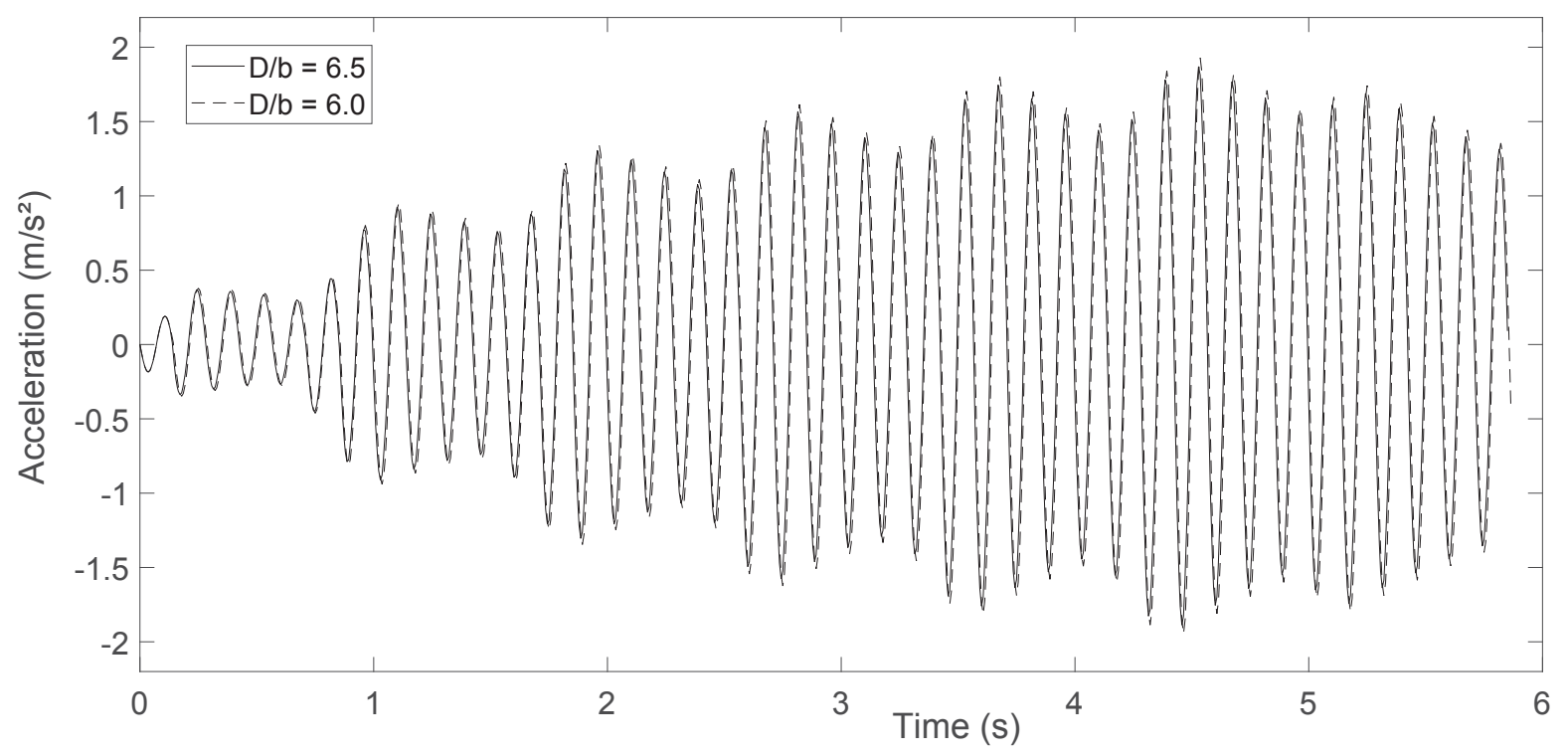

Figure 2: Time-history response of a S-S bridge under a sequence of 10 equidistant, two-axle bogies with $D=$ $18 \mathrm{~m}$, and $P=170 \mathrm{kN} . L=15 \mathrm{~m}, n_{0}=7.0 \mathrm{~Hz}, m=12 \mathrm{t} / \mathrm{m}, \zeta=1.0 \% . b=3 \mathrm{~m}$ for the dashed line; $b=2.769 \mathrm{~m}$ for the solid line.

should probably be somewhat higher for a bridge of such span, but the vibration effects will be better illustrated with the selected value.

Figure 2 shows a response that is clearly resonant, with six cycles between maximum peaks as expected from equation (3). Two time series are displayed: the dashed line corresponds to a slightly higher response, obtained with $b=3 \mathrm{~m}$, so that $D / b=6.0$; conversely, the solid line has been obtained with $b=2.769 \mathrm{~m}$, which leads to $D / b=6.5$. It should be noticed that in the latter case, the $D / b=6.5$ ratio is as far as possible from being integer, but still the observed resonant response is almost identical in both cases. So the following question arises: From the point of view of bridge vibrations, is it reasonable to consider that ATs where $D / b=D / d_{B A}$ is close to integer are more critical than others? This question is dealt with in greater detail in the following sections.

\section{NONDIMENSIONAL APPROACH BASED ON TRAIN SIGNATURE AND BOGIE FACTOR}

\subsection{Signature of a set of equidistant loads}

Based on superposition of effects in linear regime, in reference [2] the following approach was presented for the computation of the acceleration level in a S-S bridge due to the passage of a train:

$$
a_{\max }=\Gamma_{\max }(m L, \zeta, K) \cdot G\left(F_{i}, d_{i}, \lambda, \zeta\right)
$$


where the term $G\left(F_{i}, d_{i}, \lambda, \zeta\right)$ is the signature or train spectrum for a series of loads $F_{i}$ with spacing $d_{i}$ measured from the first load $\left(d_{1}=0\right)$. The wavelength $\lambda=V T$, where $T$ is the period of the vibration mode, and $\Gamma_{\max }(m L, \zeta, K)$ is the influence line for the fundamental mode of a S-S bridge. Because the influence line will vary between different types of structures, it is convenient to keep it as a separate factor, as in equation (5). In so doing, one can concentrate in the values of the signature; those values depend only on damping and the train type for each wavelength. Plots as a function of wavelength will then provide a quick view of the phenomena involved.

In reference [5], a new expression of the signature is presented for a group of $k$ equidistant, unit loads:

$$
\begin{aligned}
& G_{E}(k, \Lambda, \zeta)=k \quad \text { if } \zeta=0 \text { and } \Lambda=1,1 / 2,1 / 3, \ldots \text { (resonance) } \\
& G_{E}(k, \Lambda, \zeta)=\sqrt{\frac{\sigma^{2(1-k)} f_{k}(\sigma, \Lambda)}{f_{1}(\sigma, \Lambda)}} \quad \text { otherwise. }
\end{aligned}
$$

where

$$
\begin{aligned}
f_{k}(\sigma, \Lambda) & =\left(1+\sigma^{2 k}-2 \sigma^{k} \cos (2 k \pi / \Lambda)\right) \\
\sigma & =e^{\zeta 2 \pi / \Lambda} \geq 1 \\
\Lambda & =\lambda / D=V T / D
\end{aligned}
$$

and $f_{1}=f_{k}(k=1)$. Such expression can be computed very efficiently because it neither involves summations of out-of-phase loads, nor it involves taking the maximum among subtrains as prescribed in [2]. Conversely, it has the disadvantage that the power cars are not taken into account. The nondimensional wavelength $\Lambda$ represents the fraction of $D$ travelled in one period of the free vibration.

In order to get the complete signature for a series of carriages supported on bogies as it is usual in an AT, one has to multiply the signature given in equation (6) by the bogie factor or bogie spectrum. This factor is also introduced as a function of a nondimensional wavelength in reference [5], as explained next.

\subsection{Bogie factor}

The bogie factor is a multiplying function that can vary in the interval $[0,2]$, and provides an exact, equivalent amplitude of the effect of two consecutive loads separated by distance $b=d_{B A}$. The equivalence is established in terms of the addition of the free vibrations created by each of the two loads, when they pass over a flexible structure at speed $V$ :

$$
\begin{aligned}
f_{B}(\mu, \zeta) & =\sqrt{1+e^{-\zeta 4 \pi / \mu}+2 e^{-\zeta 2 \pi / \mu} \cos (2 \pi / \mu)} \\
\mu & =\lambda / b=V T / b
\end{aligned}
$$

In the same way that $\Lambda=1 / n_{D}=1,1 / 2,1 / 3, .$. implies resonance or sub-resonance or order $n_{D}$ associated to distance $D$ (carriage resonance), $\mu=1 / n_{b}=1,1 / 2,1 / 3, .$. implies resonance or sub-resonance of order $n_{b}$ associated to distance $b$ (bogie resonance). Also, when $\mu=$ $2,2 / 3,2 / 5, .$. bogie cancellation occurs. Conversely, above $\mu=2.0, f_{B} \rightarrow 2.0$ monotonically, in a way similar to the signature in equation (6), which tends to $k F$ when $\Lambda \rightarrow \infty$ ( $F$ is the value of the constant axle loads different from unity). 
In case of bogie resonance, integer ratios $D / b=l$ would lead to

$$
\Lambda=\frac{V T}{D}=\frac{V T}{b} \cdot \frac{b}{D}=\frac{1}{n_{b}} \cdot \frac{1}{l}=\frac{1}{n_{D}}
$$

As it is apparent, $n_{D}=n_{b} \times l$ is integer and (higher order) resonance of the carriage would also be induced. For this reason, the $D / b$ integer ratios may initially be considered more aggressive, as explained in previous section.

\subsection{Combined effect of signature and bogie factor}

The signature or spectrum of a set of $k$ two-axle bogies of constant loads $F$ can be obtained exactly by multiplication of $F$ times equations (6) and (7). Such signature will represent the vibration coupling of a series of carriages of an AT, where the power cars are neglected and all carriages are assumed to have constant length $D$. Therefore, the main coupling phenomenon between $D$ and $b$ can be analysed at once.

The signature can be suitably plot as a function of $\Lambda$ and $\mu$. In such approach it is convenient to realise that any constant ratio $\eta=D / b$, will lead to a inclined line in the $[\Lambda, \mu]$ plane, since

$$
\mu=\frac{V T}{b}=\frac{V T}{D} \cdot \frac{D}{b}=\Lambda \cdot \eta
$$

Therefore, in log-log axis the lines corresponding to constant $\eta$ ratios will appear as inclined parallel lines, as shown in Figure 3. The upper limits in Figure 3 are set by considering that $n_{0}=7.0 \mathrm{~Hz}, V_{\max }=350 \mathrm{~km} / \mathrm{h}, D_{\min }=18 \mathrm{~m}$ and $b_{\min }=2.5 \mathrm{~m}$, which are all usual values for high-speed articulated trains. Damping has been neglected, which leads to maximum values of the spectrum equal to 20 , since the number of unit loads in the trainset is $2 k=20$.

In Figure 3 the three inclined lines correspond to $\eta=D / b$ equal to $5.5,6.0$ and 6.5 . It is seen that the critical point (where $\Lambda=1 / 6$ and $\mu=1$ ) reaches a maximum amplitude of 20 for $D / b=6.0$, but the amplitudes for $D / b=5.5$ and $D / b=6.5$ are very similar. This is the theoretical explanation to the very small difference in the amplitude of vibrations levels displayed in Figure 2.

The peak response in Figure 2 can be also analysed for a wider range of speeds, as it is shown in Figure 4, where sub-resonances of the carriage are visible for $n_{D}=2$ at $226.8 \mathrm{~km} / \mathrm{h}$ to $n_{D}=8$ at $56.7 \mathrm{~km} / \mathrm{h}$, except for $n_{D}=3$ that cancels exactly (indeed it cancels twice: $i=2$ in equation 4 , and also $\mu=2$ ).

All predictions from Figure 3 are confirmed in Figure 4. The highest peak for the sixth subresonance corresponds to $D / b=6$, while the other two curves give very similar response in this case, even if they are as far as possible from being integer. Besides, the peaks for the eighth, seventh and second sub-resonances are higher for $D / b=6.5$, while the peaks for the fourth and third sub-resonances are higher for $D / b=5.5$.

The conclusions of the studies presented in this section are generalised next for a wide ensemble of S-S bridges and articulated trains that cover the vast majority of cases of interest.

\section{PARAMETRIC STUDIES}

\subsection{Hypotheses and methods}

In order to confirm that integer values of $D / b$ are not necessarily more aggressive than nonintegers as regards the coupling of carriage-induced and bogie-induced vibrations, a comprehensive parametric study has been carried out with the following characteristics. 


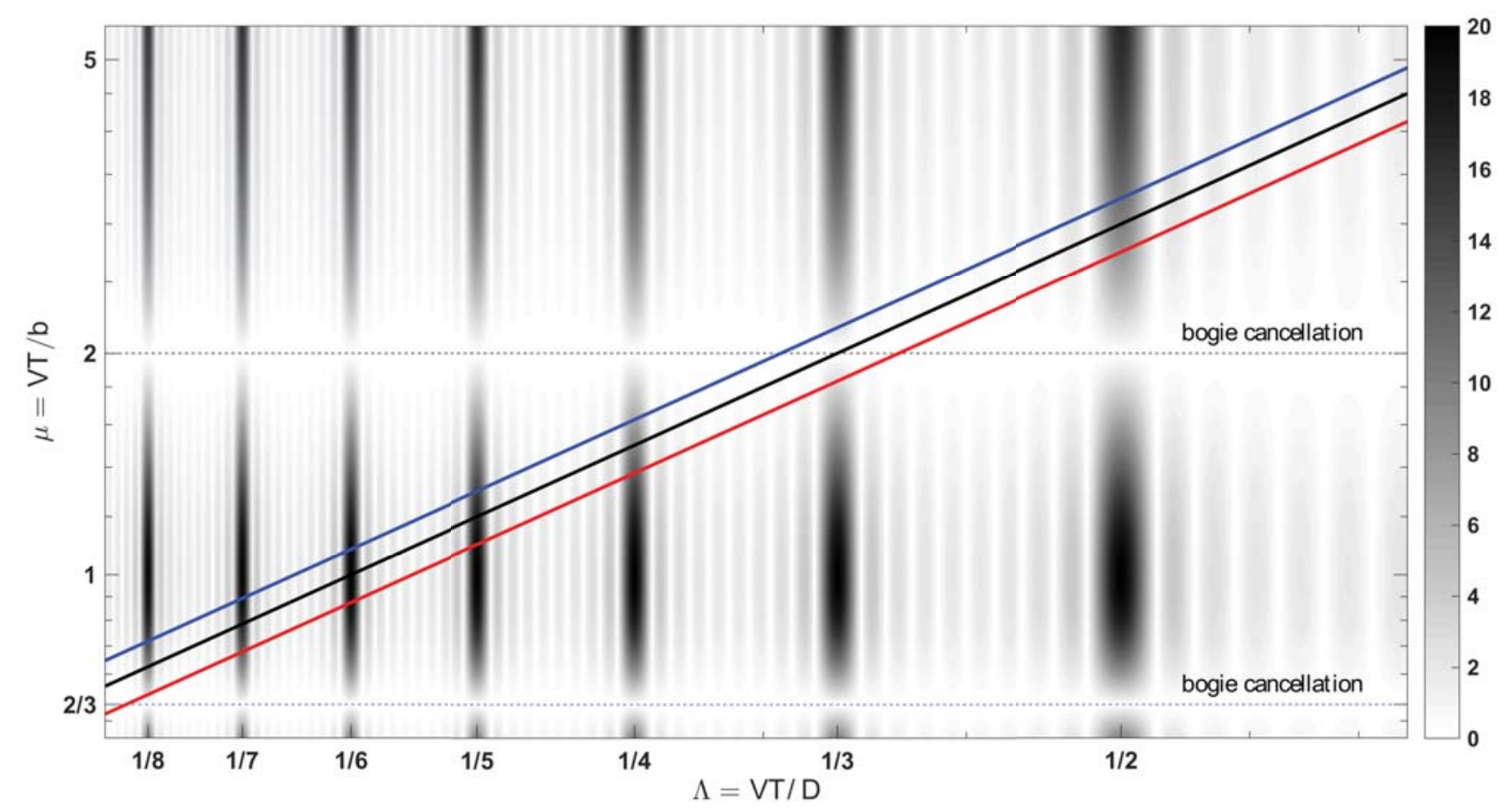

Figure 3: Signature of a sequence of $k=10$ equidistant, two-axle bogies of unit loads (log-log axis). Red, black and blue lines correspond to $\eta=D / b$ equal to $5.5,6.0$ and 6.5 , respectively.

Prestressed bridges of S-S type on rigid supports are analysed under sequences of $(10-1)=$ 9 carriages supported on equidistant bogies. Therefore, $k=10$ as in Figure 4 and each train has 20 constant, concentrated loads travelling at a constant speed $V$.

According to Table 2, the carriage length $D$ is varied in a discrete manner from $18 \mathrm{~m}$ to $27 \mathrm{~m}$ in steps of $0.5 \mathrm{~m}$, in line with the limits in Annex E from EN1991-2 [1]. Providing that the bogie wheelbase $b$ is limited to the interval $[2.5 \mathrm{~m}, 3.5 \mathrm{~m}]$ as stated also in Annex E from [1], the number of combinations of $D$ and $b$ that can produce integer ratios is limited to 51 different cases. Such cases are shown in Table 2, particularly in the two columns headed by symbol $b$; the integer $D / b=l$ value for each case is also shown in the corresponding row.

Also in Table 2, $51 \times 2$ bogie wheelbases are gathered where $D / b$ is as far as possible from being integer. For instance, in the first row of the table, $D / b=l=6$ for $D=18 \mathrm{~m}$ corresponds to $b=3 \mathrm{~m}$, while $b^{-}=3.273 \mathrm{~m}$ corresponds to a smaller ratio $D / b=6-0.5=$ 5.5. Analogously, $b^{+}=2.769 \mathrm{~m}$ corresponds to a larger ratio $D / b=6+0.5=6.5$.

It can be seen in Table 2 that some of the values of $b^{-}$and $b^{+}$lie outside the interval $[2.5 \mathrm{~m}, 3.5 \mathrm{~m}]$, but such interval is never exceeded by more than $9 \%$, so the values in the table can be deemed acceptable for the purpose of this study.

Spans from $L=5 \mathrm{~m}$ and $L=30 \mathrm{~m}$ have been analysed under the action of such $51 \times 3$ trains, and the response under the trains with integer $D / b$ values (column $b$ ) has been compared with the one due to the trains with non-integer $D / b$ values (columns $b^{-}$and $b^{+}$). Damping values for the prestressed bridges have been selected according to [1]. Clearly, the possible carriage-bogie resonant coupling situation would take place for $n_{D}=n_{b} \times l$, as explained from equation (8) in section 3.2. In such case, the resonance order for the carriage (length) is a multiple of the resonance order for the bogie (wheelbase).

If the speeds involved were to be close to $200 \mathrm{~km} / \mathrm{h}$ or higher, which is the usual range 


\begin{tabular}{|c|c|ccc||c|c|ccc|}
\hline$D / b=l$ & $D$ & $b$ & $b^{-}$ & $b^{+}$ & $D / b=l$ & $D$ & $b$ & $b^{-}$ & $b^{+}$ \\
\hline \hline 6 & 18 & 3.000 & 3.273 & 2.769 & 7 & 23 & 3.286 & 3.538 & 3.067 \\
7 & 18 & 2.571 & 2.769 & 2.400 & 8 & 23 & 2.875 & 3.067 & 2.706 \\
- & - & - & - & - & 9 & 23 & 2.556 & 2.706 & 2.421 \\
\hline 6 & 18.5 & 3.083 & 3.364 & 2.846 & 7 & 23.5 & 3.357 & 3.615 & 3.133 \\
7 & 18.5 & 2.643 & 2.846 & 2.467 & 8 & 23.5 & 2.938 & 3.133 & 2.765 \\
- & - & - & - & - & 9 & 23.5 & 2.611 & 2.765 & 2.474 \\
\hline 6 & 19 & 3.167 & 3.455 & 2.923 & 7 & 24 & 3.429 & 3.692 & 3.200 \\
7 & 19 & 2.714 & 2.923 & 2.533 & 8 & 24 & 3.000 & 3.200 & 2.824 \\
- & - & - & - & - & 9 & 24 & 2.667 & 2.824 & 2.526 \\
\hline 6 & 19.5 & 3.250 & 3.545 & 3.000 & 7 & 24.5 & 3.500 & 3.769 & 3.267 \\
7 & 19.5 & 2.786 & 3.000 & 2.600 & 8 & 24.5 & 3.063 & 3.267 & 2.882 \\
- & - & - & - & - & 9 & 24.5 & 2.722 & 2.882 & 2.579 \\
\hline 6 & 20 & 3.333 & 3.636 & 3.077 & 8 & 25 & 3.125 & 3.333 & 2.941 \\
7 & 20 & 2.857 & 3.077 & 2.667 & 9 & 25 & 2.778 & 2.941 & 2.632 \\
8 & 20 & 2.500 & 2.667 & 2.353 & 10 & 25 & 2.500 & 2.632 & 2.381 \\
\hline 6 & 20.5 & 3.417 & 3.727 & 3.154 & 8 & 25.5 & 3.188 & 3.400 & 3.000 \\
7 & 20.5 & 2.929 & 3.154 & 2.733 & 9 & 25.5 & 2.833 & 3.000 & 2.684 \\
8 & 20.5 & 2.563 & 2.733 & 2.412 & 10 & 25.5 & 2.550 & 2.684 & 2.429 \\
\hline 6 & 21 & 3.500 & 3.818 & 3.231 & 8 & 26 & 3.250 & 3.467 & 3.059 \\
7 & 21 & 3.000 & 3.231 & 2.800 & 9 & 26 & 2.889 & 3.059 & 2.737 \\
8 & 21 & 2.625 & 2.800 & 2.471 & 10 & 26 & 2.600 & 2.737 & 2.476 \\
\hline 7 & 21.5 & 3.071 & 3.308 & 2.867 & 8 & 26.5 & 3.313 & 3.533 & 3.118 \\
8 & 21.5 & 2.688 & 2.867 & 2.529 & 9 & 26.5 & 2.944 & 3.118 & 2.789 \\
- & - & - & - & - & 10 & 26.5 & 2.650 & 2.789 & 2.524 \\
\hline 7 & 22 & 3.143 & 3.385 & 2.933 & 8 & 27 & 3.375 & 3.600 & 3.176 \\
8 & 22 & 2.750 & 2.933 & 2.588 & 9 & 27 & 3.000 & 3.176 & 2.842 \\
- & - & - & - & - & 10 & 27 & 2.700 & 2.842 & 2.571 \\
\hline 7 & 22.5 & 3.214 & 3.462 & 3.000 & - & - & - & - & - \\
8 & 22.5 & 2.813 & 3.000 & 2.647 & - & - & - & - & - \\
9 & 22.5 & 2.500 & 2.647 & 2.368 & - & - & - & - & - \\
\hline & & & & & & & & & \\
\hline
\end{tabular}

Table 2: Definition of $51 \times 3$ ATs for the parametric study. 


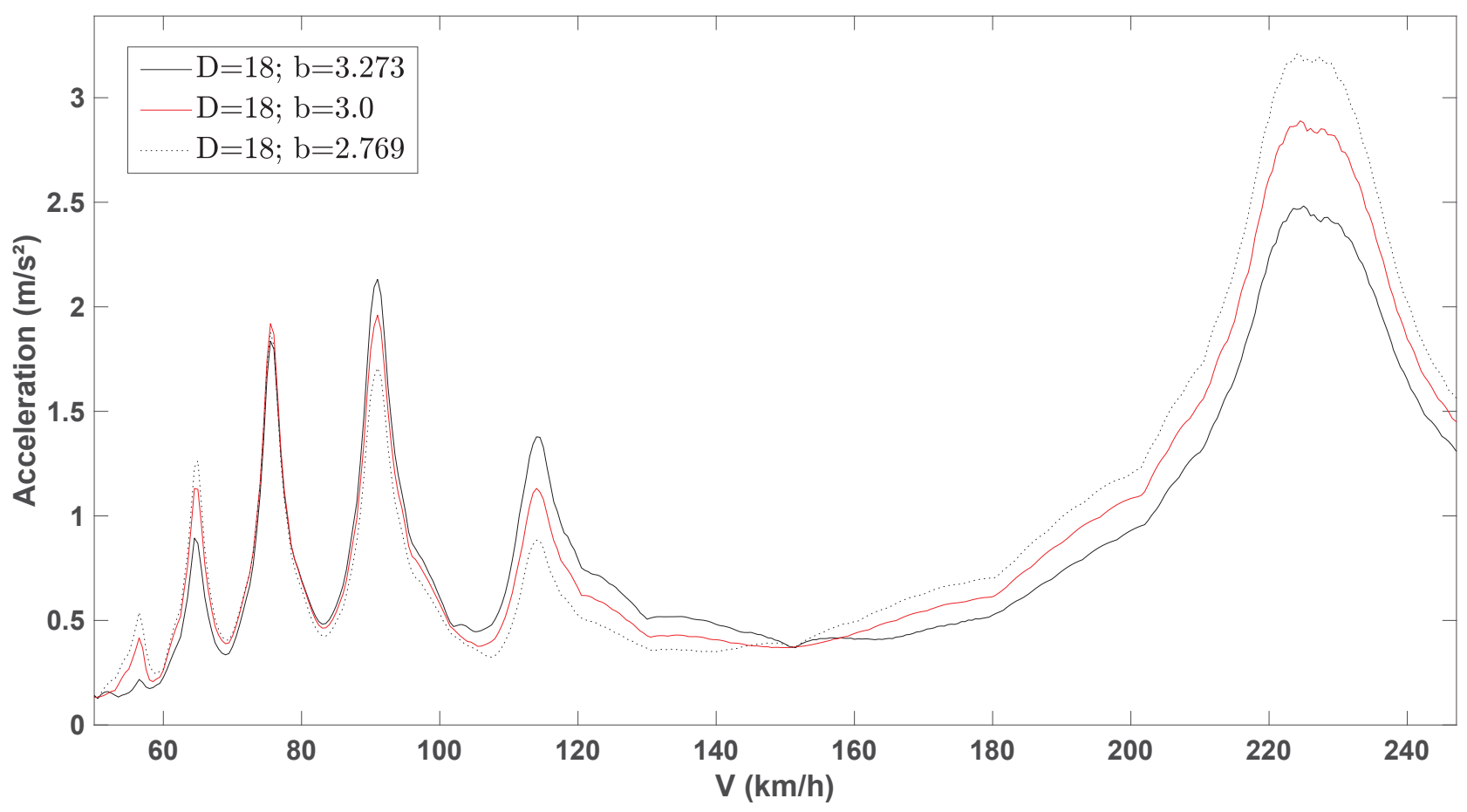

Figure 4: Acceleration envelope for a sequence of $k=10$ equidistant, two-axle bogies of $F=170 \mathrm{kN}$. Values of $b$ corresponding to $\eta=D / b$ equal to $5.5,6.0$ and 6.5 .

for dynamic analysis, $n_{b} \geq 2$ would correspond to very stiff elements and/or higher modes of frequencies above some $30 \mathrm{~Hz}$, for $b \in[2.5 \mathrm{~m}, 3.5 \mathrm{~m}]$. Moreover, $n_{b} \geq 3$ would correspond to frequencies above $45 \mathrm{~Hz}$, approximately. These are not the cases of main interest for the present study, which is focussed in the behaviour of principal structural elements. With such perspective, and given that for speeds lower than $200 \mathrm{~km} / \mathrm{h}$ vibrations have traditionally not been considered a key concern in railway bridges [6], such cases will be left out of this study; accordingly, the resonance order of the bogie will be limited to $n_{b}=1$. However, a closer nondimensional analysis such as the one presented in Figure 3 reveals that the conclusions for $n_{b}=1$ and $n_{b}=2$ are very similar.

Because resonance is a consequence of the addition of free vibrations, for a relevant resonant peak to occur the speeds of the trains must be carefully chosen in order to avoid cancellations of the influence line, as prescribed by equation 4 . Therefore, the values of nondimensional speed $K=\hat{K}_{i}$ selected for the analysis can be taken as the average ones for every two consecutive cancellations, i.e.:

$$
\hat{K}_{i}=\frac{V}{2 n_{0} L}=0.5\left(\frac{1}{2 i+1}+\frac{1}{2 i+3}\right) \quad i=1,2, \ldots
$$

Equation (10) yields nearly maximum free vibrations for each speed range between cancellations. In addition to those values $\hat{K}_{i}$, also one extra point has been selected above the first cancellation, named as $\hat{K}_{1}^{m}=0.386$ in reference [3]. Only the fundamental mode of vibration has been considered, since the addition of free vibrations for every mode obeys the same physical rules that the addition for the fundamental mode. As for the integration of the equations of motion, Duhamel's integral has been used throughout.

From previous considerations, it is clear that each train with a $D / b=l$ integer ratio will 
present maximum response when $n_{D}=n_{b} \times l=1 \times D / b$. Consequently, the sub-resonances for $\Lambda=1 / n_{D}=b / D$ from Table 2 are the ones to be analysed. This objective has been accomplished by selecting 41 different, equally spaced speed values between $\Lambda=1 /\left(n_{D}+0.5\right)$ and $\Lambda=1 /\left(n_{D}-0.5\right)$, which captures the resonant peak with the required precision.

The frequency of the bridges has been taken from the lower bound of the frequency band in section 6.4.4 from [1]. This only entails that the nominal speed depends linearly on such frequency for the analyses, but it does not affects the spans involved.

The spans are selected to avoid resonance, by using the values $\hat{K}_{i}$ obtained previously. Because sub-harmonic $n_{D}$ is the mid-point of the speed range for each analysis, then $V_{\text {target }}=$ $n_{0} D / n_{D}$ is such mid-point. Given the definition of $K$, it is straightforward to obtain the associated span for each possible $\hat{K}_{i}$, which should lie within the range $L \in[5 \mathrm{~m}, 30 \mathrm{~m}]$, or is otherwise discarded. This procedure follows the concept of the maximum resonance described in section 4 of reference [3]:

$$
L=\frac{D}{2 \hat{K}_{i} n_{D}}
$$

A total of 442 spans can be derived from the procedure explained above, which cover in a nearly continuous fashion the full range $L \in[5 \mathrm{~m}, 30 \mathrm{~m}]$, with very few minor exceptions that stem from the discretisation procedure adopted in the different ranges of parameters.

\subsection{Results and discussion}

Figures 5 and 6 summarise some of the main results of the parametric study. In each figure, the horizontal axes are merely counters of the number of different speeds (41) and number of different spans (442). The vertical axis shows the normalised maximum acceleration for every speed, obtained from time-histories of accelerations similar to the one in Figure 2.

The normalisation procedure is as follows. First, the analyses for wheelbase $b$ corresponding to $D / b=l$ integer ratios are run and, for each span $L_{k}$, the maximum resonance acceleration is computed $\left(a_{\max }^{b}\left(L_{k}\right)\right.$, where $\left.k=1,2, \ldots 442\right)$. Then, the response envelope for span $L_{k}$ is divided by $a_{\max }^{b}\left(L_{k}\right)$ and represented in Figure 5; therefore, the maximum value of all curves for every span in Figure 5 is equal to unity. Since the response values at minimum and maximum speeds display variations among different spans that render the plot too jagged, the spans have been rearranged with a view to give an appearance as smooth as possible.

Second, the analyses for wheelbase $b^{-}$corresponding to $D / b=l-1 / 2$ non-integer ratios are run and, for each span $L_{k}$, the maximum resonance acceleration is normalised against the value $a_{\max }^{b}\left(L_{k}\right)$ previously computed. In such way, the maxima of the curves for every span in Figure 6 are not equal to unity, but only slightly smaller. This phenomenon was previously demonstrated to happen for a particular case in Figure 4 (sixth sub-resonance).

From Figures 5 and 6 the comparison of maximum response values for each span has been carried out. In so doing, the conclusion is that the largest observed difference is $4.2 \%$, which is caused by the difference between $b$ and $b^{-}$. A similar comparison has also been carried out among the results corresponding to $b$ and $b^{+}$, which produces a largest difference of $3.2 \%$.

In conclusion, the parametric study conducted here shows that the peak response of sequences of equidistant bogies where $D$ is a multiple of $b$ is not significantly larger than for sequences of bogies where the ratio $D / b$ is as far as possible from being integer, when the first resonance case of the bogie $n_{b}$ is considered. 


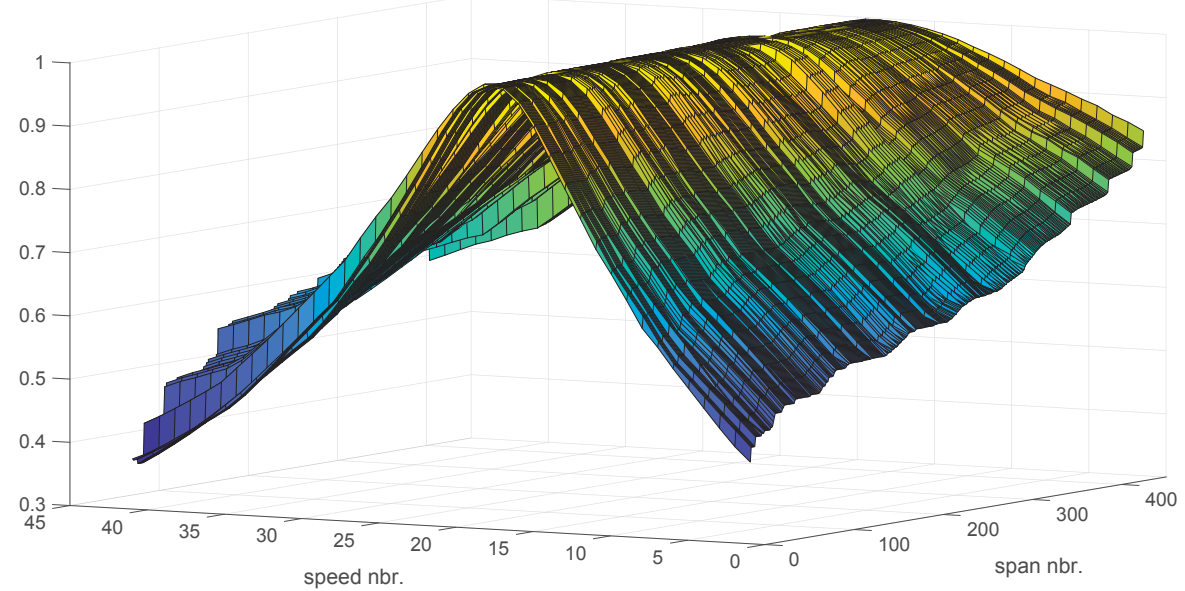

Figure 5: Envelope of maximum normalised accelerations for wheelbase $b$, corresponding to $D / b=l$ integer ratios.

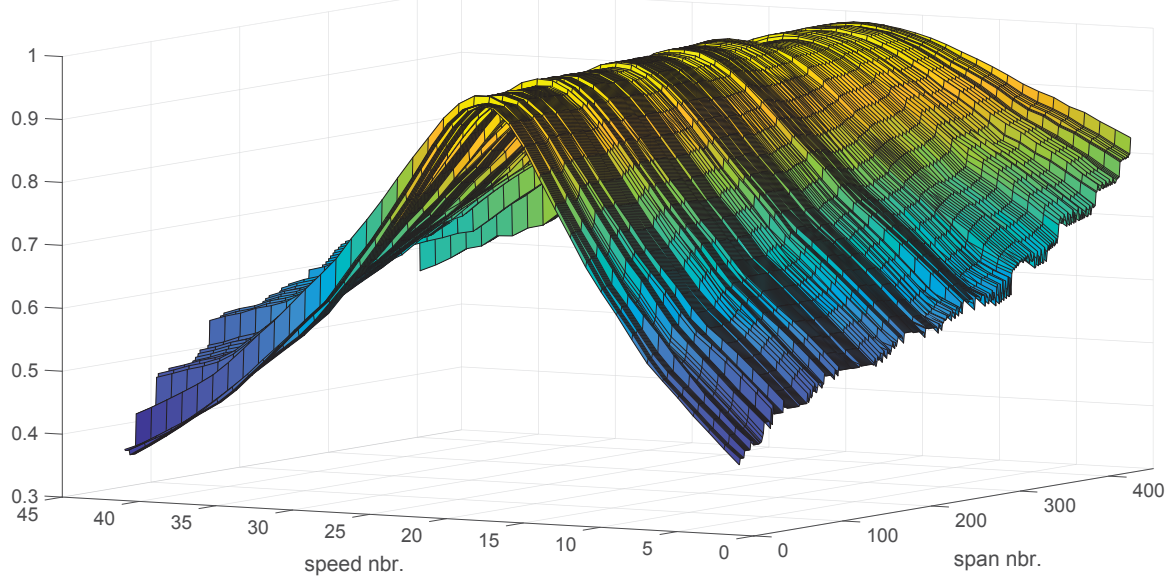

Figure 6: Envelope of maximum normalised accelerations for wheelbase $b^{-}$, corresponding to $D / b=l-1 / 2$ non-integer ratios.

\section{CONCLUSIONS}

The coupled resonance effect of sequences of equidistant Jacobs bogies has been analysed in this article. The following conclusions can be drawn from the analyses carried out in this research work:

- The addition of the free vibrations created by series of $2 k$ constant loads arranged in two-axle bogies that travel through a flexible structure can be carried out in an exact manner, by means of the multiplication of the signature (or spectrum) of a series of $k$ equidistant loads times the signature of the bogie (bogie factor). Closed-form expressions for such signatures are summarised in this paper, and were either introduced or discussed in previous works [5].

- The multiplication of the signature of a series of $k$ equidistant loads times the bogie factor yields the signature of an articulated train (power cars are neglected). Such signature can be represented in two-dimensional contour plots as a function of normalised wavelengths. 
- The two-dimensional signature of the articulated train shows that the response of a train where the ratio between carriage length $D$ and bogie wheelbase $b$ is an integer value $l$ is critical for sub-resonance order $1 / l$, in case of first resonance of the bogie wheelbase.

- Both from a theoretical perspective as well as from a comprehensive parametric study, it has been shown that actual articulated trains (as described in Annex E from EN 1991-2) are not significantly more critical when $D / b$ takes integer values than if such ratio is noninteger. This situation has been examined thoroughly for the first resonance of the bogie wheelbase.

- A closer analysis to the two-dimensional signature of the articulated train demonstrates that the preceding conclusions are also valid for the second resonance of the bogie wheelbase, which takes place at half the speed of the first one.

\section{ACKNOWLEDGEMENTS}

This research was partially developed during a stay performed by Pedro Museros at the KTH Royal Institute of Technology, within the Division of Structural Engineering and Bridges (Stockholm, Sweden). The financial support of the Generalitat Valenciana, through the program BEST2019 for research stays (Subvenciones para estancias de personal investigador doctor en centros de investigación radicados fuera de la Comunitat Valenciana), as well as the permission obtained from the Universitat Politècnica de València to carry out such stay, are gratefully acknowledged.

\section{REFERENCES}

[1] European Committee for Standardisation (CEN). EN1991-2. Eurocode 1: Actions on structures. Part 2: General Actions - Traffic Loads on Bridges. September 2003.

[2] European Rail Research Institute (D-214 Committee, ERRI D-214), Rail bridges for speeds $>200 \mathrm{~km} / \mathrm{h}$. Calculations for bridges with simply supported beams during the passage of a train (RP6), Technical Report, 1999.

[3] P. Museros, E. Moliner, L. Martínez-Rodrigo, Free vibrations of simply-supported beam bridges under moving loads: Maximum resonance, cancellation and resonant vertical acceleration, Journal of Sound and Vibration, 332, 326-346, 2013.

[4] P. Museros, E. Moliner, Comments on Vibration of simply supported beams under a single moving load: A detailed study of cancellation phenomenon by C.P. Sudheesh Kumar, C. Sujatha, K. Shankar [Int.J.Mech.Sci.99(2015) 4047,doi:10.1016/j.ijmecsci.2015.05.001], International Journal of Mechanical Sciences, 128-129, 709-713, 2017.

[5] P. Museros, A. Andersson, V. Martí, R. Karoumi, Dynamic behaviour of bridges under critical articulated trains: Signature and bogie factor applied to the review of some regulations included in EN 1991-2, Journal of Rail and Rapit Transit, (submitted for publication), 2020.

[6] European Rail Research Institute (D-214 Committee, ERRI D-214), Rail bridges for speeds $>200 \mathrm{~km} / \mathrm{h}$. Final report (RP9), Technical Report, 1999. 